\title{
PAPEL DEL ORIENTADOR/A EDUCATIVO COMO ASESOR/A: FUNCIONES Y ESTRATEGIAS DE APOYO
}

\author{
ROLE OF THE EDUCATIONAL COUNSELOR AS AN ADVICER: FUNCTIONS AND \\ STRATEGIES TO SUPPORT
}

\author{
Víctor M. Hernández Rivero ${ }^{1}$ \\ Yénifer Mederos Santana \\ Universidad de La Laguna, España
}

\section{RESUMEN}

En este artículo se analiza la función asesora del orientador/a educativo y su labor de apoyo al profesorado, es decir, se indaga sobre las características del papel que desempeña en su trabajo cotidiano con los docentes para identificar las funciones y actividades, así como los modelos y estrategias de asesoramiento que emplea. Para ello se desarrolla una investigación de corte cualitativo y se elige, entre los posibles diseños, el estudio de casos porque permite aproximarse al contexto real de los centros educativos en los que cobra importancia la figura de los orientadores. Se han analizado tres casos, cada uno de ellos con un perfil y características distintivas, adoptando criterios como la formación académica, las características del centro, la antigüedad en el puesto y el modelo de trabajo. A través de entrevistas semiestructuradas (individuales a los orientadores y grupales al profesorado), se lleva a cabo un proceso de análisis de contenido. Los resultados evidencian que estos profesionales asumen cada vez con mayor intensidad funciones de asesoramiento aunque complementariamente con funciones más

1 Correspondencia: Víctor M. Hernández Rivero: Universidad de La Laguna. Dpto. Didáctica e Investigación Educativa. Facultad de Educación. Campus Central, Avda. Trinidad s/n, 38200, La Laguna, Tenerife, Canarias. Correo-e: vhernan@ull.edu.es 
arraigadas, como la valoración psicopedagógica. Otros resultados apuntan a que existe heterogeneidad de demandas y actuaciones en los casos estudiados y que los estilos o modelos de intervención son diversos. Las conclusiones inciden en que la identidad del rol del orientador y las características contextuales determinan, en gran medida, el estilo o modelo de trabajo, que puede ser de tipo intervencionista, facilitador o colaborativo.

Palabras claves: asesoría de grupo, orientación escolar, investigación cualitativa, estudio de casos, educación

\section{ABSTRACT}

This article analyzes the advisory function of school counselors and their task of supporting teacher counseling. In other words, it looks into the characteristics of the role they carry out in their daily routine together with teachers, in order to identify the specific functions and activities, as well as the models and advising strategies used. For this purpose, a qualitative investigation is developed, choosing case study among other possible designs, since it allows an approximation to the real context of the educational centers, in which school counselors play an important role. Three cases have been analyzed, each one with its own profile and distinctive features, by adopting criterion such as academic education, features of the center, seniority and model of work. By using semi-structured interviews (individual ones for counselors and group ones for the teaching staff), a content analysis is carried out. The results show that these professionals increasingly assume more counseling functions, although complementary to other functions of a more deep-rooted nature, such as psycho-pedagogical assessment. Other results reflect the existing heterogeneity of demands and performances in the studied cases and that the styles or intervention models are diverse. The conclusions affect the identity of the role of school counselors and, to a large extent, the contextual features determine the style or model of work, which may be interventionist, facilitator or collaborative.

Key Words: group counseling, educational guidance, qualitative research, case study, education.

\section{Introducción}

La evolución del campo de la Orientación Educativa constituye una sucesiva ampliación de modelos y procedimientos de intervención para mejorar la práctica educativa (CIDE, 2009; Santana, 2014; Vélaz de Medrano, 2008). Nadie duda que la labor orientadora y el rol del orientador escolar hayan experimentado un importante desarrollo en los últimos tiempos, como respuesta a las nuevas demandas de centros y profesores, frente a los retos de los nuevos tiempos.

A lo largo de este proceso el surgimiento de modelos de trabajo de naturaleza colaborativa incorpora con fuerza al perfil del orientador/a la función de apoyo y asesoramiento al profesorado. La reformulación y ampliación de las funciones de estos agentes ha conducido al desarrollo de funciones de tipo consultivo para apoyar al profesorado ante los retos que plantea el currículo (Cholewa et al., 2017). La intervención directa sobre el alumnado se torna indirecta, para incidir preferentemente sobre el docente y la institución en su conjunto (Domingo y Fernández, 2014). 
Ahora, la relación orientador-profesor cobra mayor protagonismo, amparada en modelos colaborativos cuya finalidad lo constituye la mejora de la escuela. La figura del orientador como asesor cobra sentido bajo un papel clave en los nuevos escenarios que promueven tanto la normativa como la práctica institucional a nivel educativo en los tiempos que corren (González y García, 2007; Martínez, Krichesky y García, 2010).

Básicamente, la función de asesoramiento se construye en la interacción y comunicación bidireccional con el profesorado, buscando facilitar su labor ante los problemas que surgen en la práctica, y que tiene que ver más con la discusión y análisis conjunto de problemas que con la intervención aislada de un experto que los soluciona (Domingo, 2010a). La principal meta de este asesoramiento es que cada profesor y centro desarrollen procesos orientados y con sentido en aras a alcanzar la mejora educativa.

Aciego, Álvarez y Muñoz (2005), Lago y Onrubia (2011), y Calvo, Haya y Susinos (2012) afirman que los orientadores podrían ocupar un papel de gran importancia en los centros como agentes asesores ligados a los procesos de mejora. El asesoramiento se configura como un proceso de construcción que implica más 'trabajar con' que 'intervenir sobre', y acarrea en última instancia, un replanteamiento de las actividades que han caracterizado el rol del orientador/a.

En el siglo XX se producen numerosos intentos de integrar los servicios de orientación en los centros escolares y se lleva a cabo una intensa labor legislativa que permite mejorar la atención y orientación a la comunidad educativa. En España se adoptan diversas decisiones políticas a nivel educativo en general, y en el campo de la Orientación educativa en particular, siendo el principal referente la LOGSE y los decretos que la desarrollan, momento en el que la Orientación educativa consolida y amplía su campo de actuación, dando cabida al asesoramiento al centro como función relevante (CIDE, 2009).

En el siglo XXI la orientación educativa ya está inmersa plenamente en el ámbito escolar, pero la evolución del sistema educativo lleva a la revisión de los modelos institucionales que se proponen, complementando unos con otros, buscando unificar criterios y, sobre todo, buscando fórmulas de relación profesional para redefinir el papel del orientador, ante los nuevos retos de la enseñanza (Yáñez, 2008).

La normativa constituye en este proceso un referente principal y lleva consigo la idea de que es necesario un equipo de apoyo especializado en los centros educativos y, aunque de modo difuso, se configura el nuevo perfil del orientador/a y la reconstrucción de su labor de manera formal. No obstante, las funciones asignadas a los orientadores son motivo de constante controversia e incesante debate (Hernández, Santana y Cruz, 2007). En torno a ellas continúan las disconformidades ya que se producen en un complejo entramado de situaciones, programas, acciones y escenarios, todos ellos importantes y con frecuencia urgentes, que bloquean en no pocas ocasiones las posibilidades de flexibilidad o las opciones de priorización que darían racionalidad, integridad y cohesión a su tarea (Domingo y Fernández, 2014; Santana, 2010).

Analizar la labor asesora de los orientadores en los centros escolares, conocer de cerca su quehacer como apoyo de otros, como facilitadores de la innovación, identificando funciones, tareas y estrategias de apoyo profesional constituye el objeto de esta contribución.

\section{Modelos y estrategias de asesoramiento}

A partir de la investigación en el campo de la Organización de Instituciones Educativas y en la Teoría del Cambio en Educación, es posible identificar modelos de asesoramiento que permiten vislumbrar pautas de actuación del orientador/a como apoyo al profesorado (Domingo, 2012). 
Los modelos a los que nos referimos vienen definidos, por una parte, por el tipo de conocimiento y experiencia de las partes implicadas en relación con el contenido de los problemas que se abordan en el asesoramiento y, por otra parte, por la estructura de interacción que se construye entre asesor y asesorado y que regula el propio proceso de asesoramiento, y por ende, el de la orientación (figura 1).

Figura 1. Elementos de tipificación del modelo de asesoramiento

\begin{tabular}{|l|c|c|}
\hline $\begin{array}{l}\text { Conocimiento- } \\
\text { experiencia del profesor }\end{array}$ & MODELODE FACILITACIÓN \\
\hline $\begin{array}{l}\text { Conocimiento- } \\
\text { experiencia del asesor }\end{array}$ & MODELO DE INTERVENCIÓN & MODELO DE COLABORACIÓN \\
\hline $\begin{array}{c}\text { Elementos de tipificación } \\
\text { Relaciones dominadas por el } \\
\text { ASESOR }\end{array}$ & $\begin{array}{c}\text { Relaciones dominadas por el } \\
\text { PROFESOR }\end{array}$ \\
\hline
\end{tabular}

Fuente: Domingo, 2012

De esta forma, se identifican los siguientes patrones de actuación del orientador/asesor:

a. Modelo de intervención: El orientador/a como experto conocedor de una materia, o área de actividad es quien determina la problemática y la solución de acuerdo a su concepción de la situación. Se centra en interpretar y enjuiciar el problema, decidiendo qué se debe hacer, dedicando la mayor parte del tiempo a identificar el problema y a determinar y controlar las acciones que se deben desarrollar. Aquí se configura el rol con un estilo netamente directivo, en el que prima la intervención directa como respuesta.

b. Modelo de facilitación: Se entiende el proceso de asesoramiento como un acto de apoyo o ayuda que busca favorecer el desarrollo de habilidades y pensamiento en el docente. El orientador/a ayuda al profesorado a clarificar aspectos de la situación a analizar y los problemas, a relacionar los aspectos teóricos con los problemas que plantea la práctica de la enseñanza mediante la reflexión conjunta, proporcionándole oportunidades para facilitar la comunicación entre el profesorado. La figura del orientador/a se define como sostén de la innovación en un plano estratégico, y prima la ayuda ante los proyectos en lugar de la intervención directa sobre el problema.

c. Modelo de colaboración: En este caso las experiencias y conocimientos son compartidos, de modo que la toma de decisiones se ejerce con igualdad entre el orientador/a y el profesorado. Tanto la definición del problema como la solución es compartida por ambos, y el orientador en este entorno de trabajo conjunto acepta los conocimientos y experiencias del profesorado y viceversa, por lo que el poder y la toma de decisiones no reside en el experto sino en ambos (Odegard-Koester y Watkins, 2016). La clave ahora es 'trabajar con', potenciar y favorecer el empoderamiento profesional y de la organización. El rol que juega el orientador constituye una pieza clave como estímulo para la transformación de las prácticas educativas y las finalidades sociales. Juega un papel de liderazgo político. 


\section{Funciones de asesoramiento de los orientadores}

Entre las funciones de los asesores y, en consecuencia, del orientador/a, pueden ser identificadas las siguientes (Boza, Toscano y Salas, 2007): ayudar al profesorado en el diseño y desarrollo curricular; analizar y valorar las necesidades con el profesorado; planificación escolar a nivel organizativo y educativo; implementación y seguimiento de proyectos de innovación educativa.

También, la facilitación de la labor docente, diseminación de conocimiento e información. Evaluación formativa con feed-back como apoyo al centro educativo.

Ejerce como formador in situ del profesorado, potenciando la capacitación del profesorado mediante la ayuda y el estímulo para que desarrolle nuevas habilidades, y se dote de estrategias para la innovación curricular. A este nivel, se debe destacar su labor actuando bajo un modelo de colaboración y facilitación del cambio, como función de ayuda para la creación de estructuras de trabajo cooperativas tanto en el propio centro como con otros centros.

En definitiva, la función asesora en el ámbito de la Orientación trataría de ayudar a los docentes a introducir mejoras en las aulas y en los centros, apoyando a éstos en procesos de innovación metodológica para que la práctica docente sea más eficaz, integradora y educativa (Lago y Onrubia, 2011), y en tareas de diagnóstico institucional, planificación de la mejora e implementación del cambio organizativo. No se trata de que el orientador/a aporte soluciones elaboradas a problemas particulares -como técnico especialista- sino que actúe de facilitador en procesos para que la escuela identifique y explore lo que hace y así se encamine hacia la mejora.

Así pues, este perfil requiere una formación muy específica que le permita el desarrollo del conjunto de funciones y tareas (Aguaded y Aguaded, 2011). No obstante se debe tener presente el carácter particular, dinámico y complejo de la realidad social y, en particular, la educativa, por lo que definir modelos y patrones de actuación en relación a la función de asesoramiento constituye un reto que no está carente de dificultades y posibles contradicciones si lo que se observa es la realidad.

\section{Método}

La finalidad de esta investigación se focaliza en el análisis de la función asesora del orientador/a educativo/a y su labor de apoyo al profesorado, es decir, conocer las características del rol y su trabajo cotidiano con los docentes, identificando y definiendo las funciones y tareas que realiza, así como los modelos y estrategias de asesoramiento que emplea.

De manera más específica han sido formulados los siguientes objetivos y preguntas:

- Conocer las funciones, tareas y actividades que realiza el/la orientador/a en los centros educativos:

¿Cuáles son las principales tareas que realiza?

¿Cuáles son las tareas que desempeña como asesor del profesorado?

¿En esta relación, qué actividades son las más habituales?

¿Sobre qué temas, problemas y situaciones interviene el orientador como apoyo al profesorado? 
- Analizar qué estrategias y modelos de actuación emplea cuando lleva a cabo este tipo de intervención:

¿Qué procedimientos y estrategias de actuación emplea el/la orientador/a con el profesorado cuando le asesora?

¿Cuáles son las expectativas del orientador con respecto al profesorado en el proceso de innovación?

¿Cómo interviene el orientador/a para crear y sostener espacios de colaboración?

¿Cuál es el modo de decidir y proponer soluciones para los problemas que surgen?

\section{Tipo de estudio}

Para dar respuesta a este conjunto de interrogantes que se encuentran inmersos en una realidad social y que son fruto de interacciones entre las personas que pertenecen a la comunidad educativa, se ha optado por realizar una investigación de corte cualitativo (Álvarez y San Fabián, 2012).

Se elige el estudio de casos porque se pretende conocer detalladamente el contexto y la labor desarrollada. Según Stake (2005: 11), "el estudio de casos es el estudio de la particularidad y de la complejidad de un caso singular, para llegar a comprender su actividad en circunstancias importantes".

\section{Los casos de estudio}

Se seleccionaron tres casos, cada uno de ellos correspondientes a un orientador en un centro educativo. Los criterios de selección atienden tanto a la diversidad en la tipología de los centros (CEIP O IES), la trayectoria académica y profesional del orientador educativo, la etapa educativa en la que interviene, así como la disponibilidad y accesibilidad de los profesionales y el propio centro educativo a ofrecer la oportunidad de investigar en la institución:

- Caso A: Orientador Licenciado en Psicopedagogía, que tras 3 años desarrollando una actividad basada principalmente en la intervención individualizada y directa en su propio gabinete psicopedagógico, actualmente ejerce como orientador educativo (desde hace cuatro años) en un pequeño colegio privado concertado de educación Infantil, Primaria y Secundaria, con unos 200 alumnos/as aproximadamente. El centro cuenta con 22 profesores que conforman el Claustro.

- Caso B: Orientadora con más de 20 años de experiencia, Licenciada en Pedagogía. Desde hace dos años trabaja en un IES con 762 alumnos/as. Se trata de un centro público caracterizado por la heterogeneidad de su alumnado, atendido por un equipo de 59 profesores/as, la mayoría estable.

- Caso C: En este caso se trata de un orientador con un gran bagaje en el ámbito del asesoramiento, la investigación educativa y la enseñanza, con más de 20 años de experiencia profesional. Se caracteriza porque aporta al IES proyectos y objetivos para el trabajo conjunto de toda la comunidad educativa. La institución es de tamaño grande y acoge a 600 alumnos. 


\section{Instrumentos}

Para recabar información basada en percepciones y experiencias de los sujetos se ha elegido la entrevista de tipo semiestructurada (Jiménez, 2012). Dado que no solo se pretende conocer la visión del profesional asesor, sino también de los asesorados (profesorado), se realizó en cada centro una entrevista grupal, a modo de grupo de discusión, lo que permitió generar un debate en torno al tema establecido con un grupo que se conocen y comparten una misma realidad (Barbour, 2013).

Las entrevistas se estructuraron con el siguiente guion: un primer apartado en el cual se recoge información descriptiva, tanto del perfil profesional como académico y las características de los centros; los dos apartados siguientes mantienen consonancia con las dimensiones de la investigación, de modo que, en un segundo apartado las cuestiones se enfocan a conocer las actividades, tareas y funciones del orientador del centro y, en la tercera y última parte, las estrategias y el modelo de asesoramiento que emplean principalmente.

Las entrevistas grupales al profesorado permitieron aprovechar la dinámica que se genera en cada contexto para hacer emerger los procesos de análisis y construcción conjunta, mediante estrategias de confrontación, oposición y divergencia con el objetivo de conocer y explicar cómo los individuos perciben una determinada realidad -en este caso el asesoramiento ofrecido por el orientador del centro educativo al que pertenecen-, qué piensan y sienten ante ella.

Para contrastar la información obtenida en estas entrevistas (opiniones, visiones y experiencias de los sujetos) se lleva a cabo el análisis de documentos, principalmente del Plan de Orientación y Tutoría, las Memorias de trabajo del Dpto. de Orientación, y el Proyecto Educativo del centro, para identificar en ellos las características formales de la labor orientadora, principalmente objetivos, contenidos y actividades propuestas.

\section{Procedimiento}

El estudio se desarrolló en base a distintas fases y tareas de investigación, a lo largo de un curso escolar (2014-15). En primer lugar, se formularon los objetivos y preguntas de investigación y se procedió a la selección de casos.

Posteriormente se realiza un contacto inicial con los profesionales objeto de los diferentes estudios de casos y los centros educativos en los que trabajan, estableciendo un vínculo directo tanto con el orientador como con el profesorado. Concretados el lugar y fecha de las sesiones con cada orientador y con el profesorado, se procedió a la formación voluntaria de los grupos de debate.

Posteriormente para lograr una mayor adaptación metodológica y de los instrumentos (guías de entrevista) se realizó una recogida de datos de cada uno de los casos, mediante el análisis documental, para de este modo tener un conocimiento más exhaustivo de sus características. La información previa en este caso hace referencia a la normativa y legislación reguladora (decretos, órdenes, circulares, etc.); los Proyectos Educativos y las Memorias de los centros, así como a los planes de trabajo y memorias que con carácter interno los Departamentos de Orientación elaboran para facilitar la coordinación, el seguimiento y la valoración de sus actuaciones.

Se realizaron entrevistas individuales a los orientadores, y grupales al profesorado. De este modo, la recogida de datos se distribuyó en tres entrevistas a orientadores de centros, y dos sesiones de debate mediante entrevistas grupales a 1 grupo de profesorado en cada uno de los casos estudiados (en total 3 entrevistas grupales con dos sesiones de debate cada una). En total participaron 3 orientadores/as y un total de 24 profesores/as pertenecientes a 2 centros educativos 
de Educación Infantil y Primaria, y 1 centro de Educación Secundaria, constituidos en 3 grupos de 8 miembros cada uno.

\section{Análisis e interpretación de la información}

Para llevar a cabo el análisis de los datos obtenidos en las diferentes entrevistas se seleccionó, de entre los diversos procedimientos existentes, el denominado análisis de contenido a través de matrices. Supuso realizar un estudio exhaustivo de la información para determinar sus partes, la relación entre ellas y con el todo.

Se registró la información de forma literal y se codificó y categorizó, asignando códigos o abreviaturas al texto generado, de modo que los datos quedaron agrupados por similitud de contenido o idea expresada, facilitando su comprensión y el manejo de la información.

Por último, para lograr agrupar los datos de un modo comprensible y útil, se elaboraron matrices de acuerdo a cada una de las dimensiones de análisis.

Tras realizar un estudio exhaustivo agrupando la información en unidades elementales, comprensivas y relevantes se organizaron los resultados como construcción conceptual.

\section{Resultados}

Para la presentación de resultados se ha optado por recoger de manera ordenada y resumida la información de cada caso en tres Tablas, en las que puede consultarse una síntesis de los datos obtenidos para cada una de las dimensiones de estudio (Tablas 1, 2, y 3).

Al mismo tiempo se ha intentado analizar los casos comparativamente, para lograr una visión de conjunto, aunque con las limitaciones que imponen la investigación cualitativa y la idiosincrasia de cada contexto y caso.

\section{Actividades, tareas y funciones de asesoramiento del orientador/a}

En los tres casos destaca la gran cantidad de funciones y tareas que se le atribuye al orientador, lo que sin lugar a dudas constituye una muestra de la amplitud y complejidad de su rol. Su intervención se produce con todos los agentes de la comunidad educativa y también con otros agentes externos para abordar distintas temáticas y problemáticas, en ocasiones vinculadas a la innovación, la formación del profesorado y la mejora institucional.

Las familias, el profesorado, el alumnado, la Administración y otros sistemas de apoyo constituyen los roles complementarios con los que se relaciona. Para los orientadores constituye un verdadero reto afrontar tal variedad de actividades y tareas, que van desde la atención directa con el alumnado hasta la mediación entre la familia y la escuela, el apoyo al profesorado, o la colaboración con los equipos directivos.

De manera externa destaca el trabajo de intercambio de información y experiencia con el EOEP de la zona y la propia Administración educativa, en un contexto que día a día es cambiante 
y presenta nuevas situaciones que requieren total atención y mucho tiempo. Así quedó plasmado en las entrevistas:

- Orientador (caso A): "Cada día mi plan puede variar, según la situación que tenga la escuela, según los niños y el profesorado, que nunca vienen igual. Yo tengo unas tareas a realizar y sé cómo actuar, pero la dinámica diaria los van cambiando."

- Orientadora (caso B): "Tengo multitud de funciones como asesora, dinamizadora y orientadora, intento planificar y seguir un programa, pero el día a día del centro con su ritmo de trabajo y su generación de vida propia, por así decirlo, genera casos que no tienes previstos y hay que atenderlos."

- Orientador (caso C): "En primer lugar, hay funciones bien delimitadas, uno hace propuestas para la elaboración del PAT y para la orientación académica y profesional y luego hay que coordinar su desarrollo; en segundo lugar hacer propuestas relativas al Plan de Atención a la Diversidad y coordinar al profesorado-tutor; éstas serían las funciones que básicamente yo desarrollo en el centro".

También son frecuentes las intervenciones cuando la actuación se realiza a nivel institucional, primando las funciones de apoyo en relación a los procesos de enseñanza-aprendizaje y con proyectos institucionales:

- Orientador (caso A): "Todas las actuaciones, adaptaciones curriculares, propuestas de trabajo etc. son elaboradas por el profesorado, el orientador lo que hace es poner un poco en función de su conocimiento o asesorarles en cómo se deben hacer las cosas, pero es trabajo del profesorado el elaborar esas cosas".

- Orientadora (caso B): "Pienso que es por nuestra formación, somos más psicopedagogos que docentes y los profesores didactas, entonces el campo del desarrollo cognitivo, los problemas familiares, el desarrollo curricular, etc. son principalmente competencia de ellos"

- Orientador (caso C): "El tema del asesoramiento organizativo y curricular para mí es clave en nuestra labor de apoyo, desde hace unos cuantos años". 
Tabla 1. Análisis de funciones realizadas y procedimientos de asesoramiento en el caso $\mathrm{A}$

\begin{tabular}{|c|c|c|}
\hline & $\begin{array}{c}\text { Actividades, tareas y funciones de asesoramiento } \\
\text { más frecuentes del orientador/a }\end{array}$ & $\begin{array}{l}\text { Estrategias habituales y modelo de } \\
\text { asesoramiento del orientador }\end{array}$ \\
\hline & $\begin{array}{l}\text { - Aportar información al profesorado para la } \\
\text { mejora de su práctica docente. } \\
\text { - } \quad \text { Ayudar a los profesores a valorar sus propios } \\
\text { materiales o prácticas, a relacionarse con } \\
\text { colegas y a crear redes de intercambio y } \\
\text { colaboración en base a intereses comunes. } \\
\text { - Capacitar al profesorado para identificar y } \\
\text { resolver sus propios problemas, planificar y } \\
\text { poner en práctica sus proyectos particulares } \\
\text { de innovación y mejora. } \\
\text { - Recopilar, sintetizar, transformar y difundir } \\
\text { información para el profesorado, mediante el } \\
\text { intercambio de nuevas ideas, materiales } \\
\text { curriculares, hallazgos de investigación, } \\
\text { nuevas prácticas. } \\
\text { Observar y seguir la actuación del profesorado } \\
\text { en el aula. } \\
\text { - Asesorar y aconsejar en las actuaciones, } \\
\text { adaptaciones curriculares, propuestas de } \\
\text { trabajo etc. } \\
\text { Observar y valorar al alumnado. } \\
\text { - Intermediar entre las familias y el profesorado. } \\
\text { Orientación y asesoramiento al profesorado en } \\
\text { las adaptaciones curriculares, atención al } \\
\text { alumnado con NEAE y NEE, y actuación con } \\
\text { las familias. }\end{array}$ & 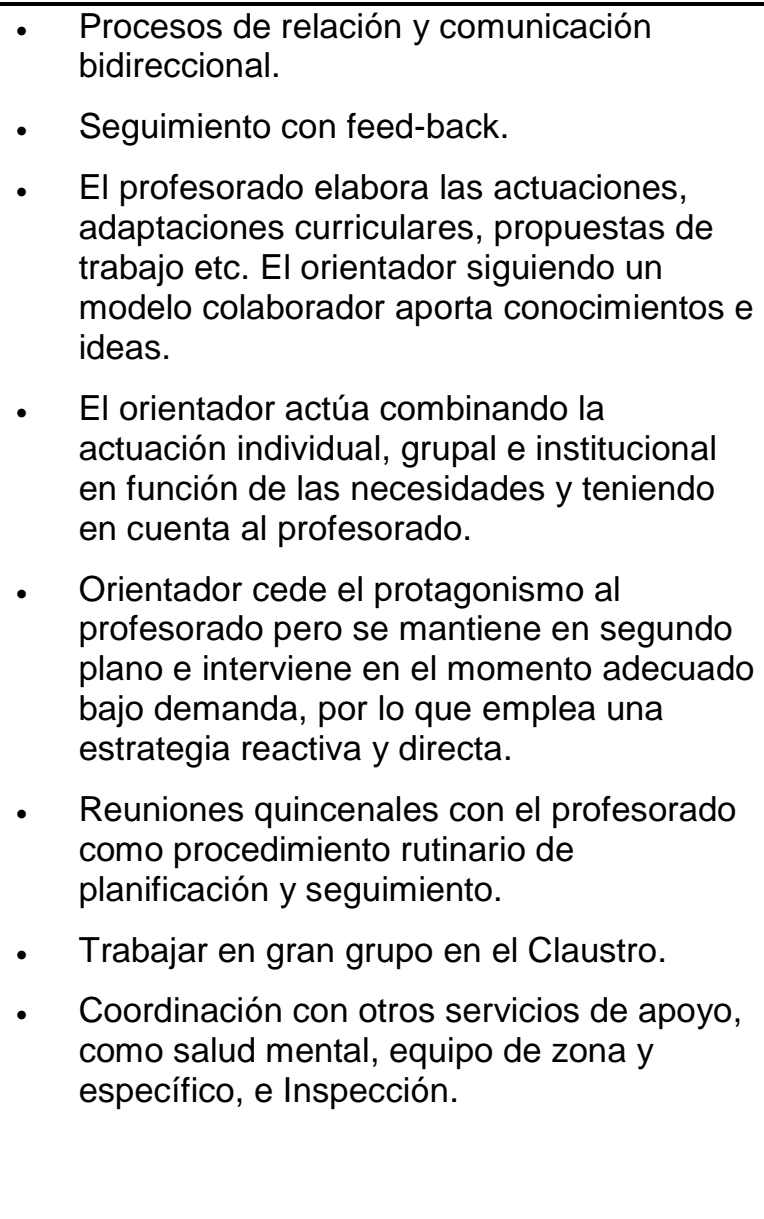 \\
\hline
\end{tabular}

Fuente: Elaboración propia

Analizados cada uno de los casos, es posible ofrecer una primera caracterización de su labor asesora al profesorado identificando funciones clave tales como ayuda, asistencia, apoyo, consejo, información, consulta, recomendación, sugerencia, capacitación, formación, supervisión, lo que convierte a esta figura en una pieza clave en los procesos educativos y de mejora en los centros educativos:

- Orientador (caso A): "El tutor detecta cualquier dificultad y el orientador tiene que ir aplicando una serie de procedimientos de observación y valoración"; "aconsejar o sugerir, apoyar y asesorar en lo que sea necesario, dándole importancia a sus aportaciones"; "el orientador lo que hace es aportar en función de su conocimiento y ayudarles en cómo se deben hacer las cosas".

- Orientadora (caso B): "Intento ser formadora de profesores a su vez que dinamizadora y asesora, creo que cuanto ellos más sepan del campo en el que están trabajando, pues más podrán sentirse seguros"; "intento facilitarles el trabajo y burocratizárselos lo menos posible"; "somos asesores, dinamizadores y orientadores" 
- Orientador (caso C): "Ofrezco herramientas a quien está a pie de aula para que pueda ser autónomo y que en un futuro pueda asumir y adquiera capacidad"; "tienes que moverte siempre en una situación dual de intervenir para resolver y de actuar como un asesor que forma a otras personas para que desarrollen su labor"; "puedo aportar materiales, mi asesoramiento y mi experiencia y los tutores lo que aportan es el conocimiento que tienen de su grupo y su alumnado".

Tabla 2. Análisis de funciones realizadas y procedimientos de asesoramiento en el caso B

\begin{tabular}{|c|c|c|}
\hline & $\begin{array}{c}\text { Actividades, tareas y funciones de asesoramiento } \\
\text { más frecuentes del orientador/a }\end{array}$ & \\
\hline & $\begin{array}{l}\text { Diseñar planes, programas, actividades y } \\
\text { propuestas de trabajo para el profesorado con } \\
\text { seguimiento y valoración de las acciones. } \\
\text { - Informar y ayudar al profesorado y al centro a } \\
\text { implementar directrices establecidas desde } \\
\text { instancias administrativas. } \\
\text { - Determinar requerimientos que deben cumplir, } \\
\text { especificar resultados o logros que se deben } \\
\text { alcanzar, establecer procesos o procedimientos } \\
\text { a seguir, etc. } \\
\text { - Realizar intercambio de información o derivar } \\
\text { casos con sistemas de apoyo externos. } \\
\text { - Poner en contacto a los centros con fuentes de } \\
\text { información y conocimiento. Transferir nuevas } \\
\text { ideas, materiales curriculares, hallazgos de } \\
\text { investigación, nuevas prácticas. } \\
\text { - Asesorar al profesorado para indagar sobre sus } \\
\text { prácticas. } \\
\text { Aporta métodos, herramientas y estrategias así } \\
\text { como facilitar dinámicas de trabajo para el } \\
\text { profesorado. } \\
\text { Orientación académica. } \\
\text { Diagnosticar e intervenir con el alumnado con } \\
\text { NAEA y NEE. } \\
\text { Intervenir con el alumnado con conductas } \\
\text { disruptivas que dificultan el transcurso de la } \\
\text { clase. } \\
\text { Programación de las sesiones de tutoría. }\end{array}$ & $\begin{array}{l}\text { - La comunicación es con frecuencia } \\
\text { unidireccional, el orientador da información } \\
\text { y el profesorado la recibe. } \\
\text { Desempeña un modelo directivo en el que } \\
\text { decide sobre qué actuar y cómo, } \\
\text { marcando las pautas para que el } \\
\text { profesorado intervenga. } \\
\text { - Estrategia reactiva con el profesorado y } \\
\text { proactiva con la institución, dado el tiempo } \\
\text { disponible. } \\
\text { - La intervención es grupal e institucional, el } \\
\text { acercamiento al orientador es a través de } \\
\text { las reuniones establecidas por ciclos y en } \\
\text { casos muy urgentes en cualquier } \\
\text { momento. } \\
\text { El orientador aporta métodos, } \\
\text { herramientas y estrategias que faciliten las } \\
\text { dinámicas de trabajo del profesorado tutor. } \\
\text { Actuación indirecta sobre el problema o } \\
\text { necesidad: trabaja con el profesorado para } \\
\text { que éste intervenga y resuelva los } \\
\text { problemas. }\end{array}$ \\
\hline
\end{tabular}

Fuente: Elaboración propia

Aun así, los centros educativos son entornos dinámicos donde el día a día presenta una nueva realidad y esto provoca que a pesar de la variedad de funciones de asesoramiento, las actividades de los orientadores estén focalizadas frecuentemente en la resolución de problemas de aula y el diagnóstico y atención al alumnado con NEAE. Así lo expresan los orientadores y así lo manifiesta el profesorado: 
Profesorado (caso A): "Nos ayuda con casos complicados de comportamiento, con casos que presentan dificultades en el aprendizaje", "En realidad nos atiende sobre todo cuando tenemos niños con NEAE."

Profesorado (caso B): "Lo más frecuente es que nos atienda para las sesiones de tutoría, es lo más habitual. También lo hace cuando existe algún alumno o alumna con necesidades especiales o problemático."

Tabla 3. Análisis de funciones realizadas y procedimientos de asesoramiento en el caso C

\begin{tabular}{|c|c|c|}
\hline & $\begin{array}{c}\text { Actividades, tareas y funciones de asesoramiento } \\
\text { más frecuentes del orientador/a }\end{array}$ & $\begin{array}{l}\text { Estrategias habituales y modelo de } \\
\text { asesoramiento del orientador/a }\end{array}$ \\
\hline & $\begin{array}{l}\text { - Asesorar y establecer pautas dentro de los } \\
\text { cauces legales. } \\
\text { - Ayudar al centro a implementar directrices } \\
\text { curriculares establecidas desde instancias } \\
\text { administrativas así como seleccionar, adaptar } \\
\text { e implementar programas disponibles que } \\
\text { responden a sus necesidades; o desarrollar } \\
\text { su propio currículo. } \\
\text { - Ofrecer las herramientas a quien está a pie } \\
\text { de aula para que pueda ser autónomo, } \\
\text { capacitarles para identificar y resolver sus } \\
\text { propios problemas, planificar y poner en } \\
\text { práctica sus proyectos de innovación y } \\
\text { mejora, para alcanzar un mayor grado de } \\
\text { autonomía. } \\
\text { Promover, planificar, conducir y supervisar } \\
\text { actividades de mejora en la escuela. } \\
\text { Diseñar planes o programas, elaborar } \\
\text { materiales, orientaciones, propuestas de } \\
\text { trabajo para que el profesorado los utilice y } \\
\text { ponga en práctica. } \\
\text { Recopilar, sintetizar, transformar, difundir } \\
\text { información para el profesorado, poniendo en } \\
\text { contacto a los centros con fuentes de } \\
\text { información y conocimiento. } \\
\text { Asesorar y solucionar situaciones de } \\
\text { alumnado con conductas disruptivas. }\end{array}$ & $\begin{array}{l}\text { - Estrategias de comunicación, negociación, } \\
\text { participación, colaboración, reflexión, } \\
\text { valoración y evaluación con el profesorado } \\
\text { - La comunicación es bidireccional. La } \\
\text { intervención indirecta, ofreciendo } \\
\text { herramientas al profesorado para que en } \\
\text { futuras situaciones pueda actuar con } \\
\text { autonomía. } \\
\text { - El orientador ofrece pautas e información y } \\
\text { la toma de decisión es conjunta con el } \\
\text { profesorado. } \\
\text { Intervención preferentemente grupal } \\
\text { mediante reuniones con tutores por ciclos y } \\
\text { en ocasiones por niveles semanales. Así } \\
\text { como institucional en colaboración con la } \\
\text { jefatura de estudios. } \\
\text { Provocar participación en las reuniones. } \\
\text { Ayudar en la exposición de las ideas. }\end{array}$ \\
\hline
\end{tabular}

Fuente: Elaboración propia

En resumen, respecto a esta primera categoría de análisis, destaca la gran cantidad de funciones y tareas de asesoramiento que desempeñan los orientadores en los centros educativos; en los tres casos estudiados existe coincidencia en aquellas destinadas a informar, aconsejar, apoyar, planificar y supervisar entre otras.

A pesar de ello, frecuentemente su actuación se relaciona con la atención a las necesidades educativas especiales, focalizada en el alumnado desde una labor de detección y valoración psicopedagógicas, y en ocasiones, de intervención directa sobre problemas de aula y de centro. 


\section{Estrategias y modelo de asesoramiento del orientador}

Ésta dimensión de análisis responde a la cuestión de cómo y mediante qué estrategias y procedimientos se asesora al profesorado y se materializa el apoyo prestado por el profesional, en el marco del contexto y de las relaciones que se producen.

Del análisis se desprende una idea clave: no existe un modelo consolidado que represente a cada uno de los procesos de asesoramiento que desarrollan los orientadores, ni un conjunto único de estrategias empleadas.

Los resultados muestran que se produce disparidad entre los casos, representando cada uno de ellos una tendencia o estilo de trabajo.

En el caso A tanto el orientador como el profesorado se sienten satisfechos con su modo de trabajar y relacionarse, siendo protagonista el profesorado en la planificación y actuación de sus acciones desde un modelo de facilitación y estilo no directivo.

Mientras, en el caso B el profesorado siente la necesidad de más autonomía y menos dirección. Se trata de un modelo técnico basado en la intervención, en el que prima la actuación del orientador y la toma de decisiones por parte de ésta.

Con respecto al caso $\mathrm{C}$ se mantiene una relación de colaboración donde ambos respetan las aportaciones del otro buscando el consenso y la ayuda mutua para la mejora colectiva. Se define claramente como un modelo colaborativo de asesoramiento en el que las estrategias de apoyo atienden preferentemente la innovación a nivel institucional, aunque tampoco se abandona la intervención grupal o individual.

De este modo quedó plasmado durante las entrevistas:

- Orientador (caso A): "Mi manera de actuar es dándole tiempo para que cuenten con sus propias estrategias. Cuando hayan agotado estas estrategias ya les asesoro, una vez hayan actuado les pido información de las actuaciones que han desarrollado y por ultimo yo busco otras"; "Con el profesor, me mantengo al margen mayormente porque es él quien quiere actuar, en muchos casos está más preparado o cree estar más preparado y quiere resolver el mismo el problema."

- Orientadora (caso B): "Yo llevo mi idea, mis conocimientos, de hecho somos expertos en el ámbito de la orientación, e intento que ellos también vean que decisión estoy pensando y que ellos planteen ideas y su opinión."

- Profesorado (caso B): "Nos dice lo que se tiene que tratar y de qué manera." "Puede que en alguna ocasión te pida una sugerencia o tú la aportes pero en general está estipulado de antemano que hacer."

- Orientador (caso C): "Depende un poco de la problemática; por lo general mi modelo de intervención tiende a ser indirecto, es decir, ofrecer herramientas a quien está a pie de aula para que pueda ser autónomo" "yo lo que tengo que hacer es llegar a la reunión clarificar los problemas y necesidades que más o menos se plantean, escuchar las necesidades y problemas que los profesores detectan y, en un proceso de reflexión y debate conjunto, ir elaborando lo que se va a hacer, hasta llegar a un punto de consenso." 
Todos coinciden en que son las reuniones de todo tipo y en todos los niveles organizativos el punto referente para el intercambio de información y el debate, no obstante, las reuniones son más frecuentes con el profesorado tutor.

Aunque están dispuestos a actuar con todo el profesorado y con una atención más personal e individualizada, este tipo de intervención se restringe debido a la escasez de tiempo:

- Orientador (caso A): "Pues mensuales o en ocasiones trimestrales, a no ser que se presente algún caso concreto que el profesor necesite apoyo y asesoramiento que es ese caso las realizo más habituales, para poder realizar un seguimiento de la intervención y cómo evoluciona el problema".

- Orientadora (caso B): "Tengo reuniones con tutores semanales, tengo reunión semanal con el equipo directivo, tengo reuniones con el EOEP, reuniones con los coordinadores de ámbito y reuniones con mi propio departamento, es la forma en que el profesorado se puede acercar por los cauces establecidos al Departamento de Orientación"; "Las reuniones son únicamente con el profesorado que es tutor".

- Orientador (caso C): "Semanalmente me voy reuniendo con los tutores de cada nivel y vamos coordinando el desarrollo del PAT. Por ejemplo las primeras semanas se desarrolla el plan de acogida, pues durante esas semanas nos reunimos orientadores y profesores para determinar qué tipo de acciones se van a hacer". "Las reuniones también se hacen por niveles, por un tema de economía de esfuerzo y horas disponibles".

En cuanto a estrategias de actuación para el asesoramiento, el orientador aprovecha las ocasiones y momentos que se presentan para crear vías de comunicación entre grupos, permitir la reflexión y análisis e interpretación de las prácticas que se desarrollan en el aula y fuera de ellas, permitiendo la participación del profesorado.

En este sentido los comentarios recogidos son:

- Orientador (caso A): "Trato de que entiendan que tenemos que trabajar en equipo".

- Orientador (caso B): "Intento pasar poco tiempo en el despacho y más tiempo en la sala de profesores, porque es donde se da la convivencia del profesorado".

- Orientador (caso C): "Es competencia de los equipos directivos y los orientadores crear espacios de debate, diálogo, de reflexión conjunta y dinamizarlos, nosotros trabajamos en colaboración con la Jefatura de Estudios"; "yo lo que tengo que hacer es llegar a la reunión plantear los problemas y necesidades que más o menos se detectan, y en un proceso de reflexión y debate conjunto ir elaborando lo que se va a hacer para llegar a un punto de consenso".

En conclusión, es cierto que los orientadores consideran al profesorado parte fundamental en el proceso de intervención, ya que posee conocimientos y experiencias útiles para la elaboración de planes, programas e intervenciones, pero no todos de igual modo, lo que marca una línea entre aproximarse más a un modelo que a otro, a un estilo más o menos directivo.

En este sentido, se detectan ciertas tendencias metodológicas y unos rasgos definitorios para el desempeño del rol del orientador/a como asesor/a:

En el caso A puede considerarse que se aproxima más a un modelo de facilitación, donde el orientador cede el protagonismo al profesorado, teniendo una expectativa de ellos alta, ya que le 
atribuye conocimientos y estrategias necesarias para actuar solo, aunque el orientador va a su lado para intervenir cuando sea requerido.

En el caso B la orientadora se identifica como experta, elaborando y programando toda la actuación, aunque permite aportaciones sobre su material que puedan mejorarlo; en este caso su mayor incidencia con el profesorado es para aportarle información legislativa, datos, propuestas etc. El profesorado se siente satisfecho pero no con las oportunidades de participación. Este efecto en el colectivo asesorado es producto de un modelo de asesoramiento de corte técnico basado en la intervención.

Por último, en el caso $\mathrm{C}$ el modelo que se vislumbra es el de colaboración, en el que el orientador concede gran protagonismo al profesorado, para aportar conocimientos y experiencias y así tomar las decisiones de forma conjunta; el asesor aprovecha los espacios para la participación conjunta y aporta sugerencias y conocimientos, respetando las relaciones de paridad escrupulosamente.

\section{Conclusiones}

En la primera categoría analizada, que hace referencia a las actividades, funciones y tareas de asesoramiento del orientador, la labor de apoyo y el asesoramiento representa un ámbito de actuación más o menos novedoso, que no se limita únicamente a la actuación con el profesorado, sino que su intervención se produce con toda la comunidad educativa en busca de la mejora del centro. Así, es una figura que detecta problemáticas del alumnado, asesora al profesorado e interviene con la familia, lo que coincide con lo encontrado por Domingo (2010b).

En cuanto a las funciones de asesoramiento más frecuentes y destacadas por los propios orientadores/as se encuentran actividades de recopilación, síntesis, transformación y difusión de información para el profesorado, poniendo en contacto a los centros con fuentes de información y conocimiento; la transferencia de nuevas ideas, materiales curriculares, hallazgos de investigación, nuevas prácticas mediante el aporte de materiales, asesoramiento y experiencia, sobre todo con el objetivo de ofrecer las herramientas al profesional que se encuentra en el aula para dotarlo de mayor autonomía; capacitarles para identificar y resolver sus propios problemas, planificar y poner en práctica proyectos de innovación y mejora, así como alcanzar un mayor grado de autonomía.

Sin embargo -y a pesar de la conciencia que se tiene con respecto a la importante labor asesora que realizan estos agentes con el profesorado y la alta incidencia que ésta tiene en la mejora del centro educativo (Calvo, Haya y Susinos, 2012; Sink, 2016)-, los análisis apuntan que entre las actividades más frecuentes se encuentran las destinadas a la detección de alumnado con NEAE y el diagnostico e intervención sobre el caso, no sólo por ser el especialista en este tema en el centro, sino porque el profesorado lo reclama con el alumnado que presenta conductas disruptivas que dificultan el transcurso de la clase y para buscar alternativas con el alumnado que, por dificultades en el aprendizaje por discapacidad o circunstancias puntuales, no pueden seguir el ritmo que se marca en el aula.

En la segunda dimensión los análisis muestran disparidad en el modelo de asesoramiento que representa a cada uno de ellos, no obstante en ninguno de los casos es definitiva su clasificación y las conclusiones de este estudio han de tomarse con cautela. La identidad de cada orientador determina el modo de asesorar, junto a otros aspectos como la cultura colaborativa del centro y 
las características institucionales y del contexto. El modelo de asesoramiento se construye en el entramado entre lo que "somos" y lo que "hacemos". Serían necesarios otros estudios que profundizaran en este aspecto.

En el estudio se han seleccionado casos en función de criterios que permiten una cierta comparación de los datos para concluir que a pesar de los requerimientos legales y de la semejanza en las funciones y tareas, cada centro escolar y las personas que en él interactúan configuran una relación de asesoramiento particular. Santana (2010: 267), en este sentido, afirma: "los orientadores han de hacer lo posible por entender la situación de los otros roles con los que se relacionan, cambiando su modo de aproximación".

Por otra parte, el tiempo disponible es un hándicap en el proceso de asesoramiento, dado que son muchas las funciones y tareas que se le atribuyen al orientador que en ocasiones se siente desbordado. El profesorado, en su mayoría, reclama más atención por parte del asesor pero, consciente de las rutinas y condiciones, aceptan las reuniones como el momento clave de compartir.

Sin embargo, el estudio realizado a partir del análisis de estos tres casos presenta algunas limitaciones desde el punto de vista del tamaño (número de casos estudiados) y el ámbito geográfico (circunscrito a la realidad de Canarias). En este sentido sería recomendable realizar otros estudios en esta dirección que permitieran profundizar tanto en su perfil como las circunstancias en que se desenvuelve. Algunas otras limitaciones se derivan de la instrumentación empleada. Sugerimos para futuras investigaciones ampliar el tipo de instrumentos para la recogida de información, tales como la observación y la estancia en el campo, lo que permitiría conocer con mayor detalle y profundidad la realidad del trabajo de la orientación en los centros, evitando el sesgo que conlleva obtener datos y conclusiones a partir de las propias percepciones y opiniones de los sujetos (entrevistas). Nos hemos encontrado con un marco profesional difuso con complejas condiciones institucionales y de trabajo que requieren técnicas de estudio diversas y complementarias.

Para terminar, se ha de resaltar la importante figura de los orientadores en los centros como asesores y apoyo del profesorado. La evolución tan dinámica que se produce en el sistema educativo y los procesos de enseñanza-aprendizaje necesitan de este tipo de profesionales en el centro para que ayuden al profesorado a elaborar proyectos, resolver situaciones de aula y centro, formarse y adquirir capacidades, en colaboración con sus compañeros de trabajo.

Es necesario seguir investigando este rol para comprender un perfil a todas luces imprescindible y relevante para la mejora educativa, que requiere de una formación y especialización decididamente enfocada a la innovación (Aguaded y Aguaded, 2011). En este sentido sería recomendable apoyar mucho más la formación, coordinación y toma de decisiones sobre esta labor en base al análisis de la propia práctica y a los resultados de la investigación.

\section{Referencias Bibliográficas}

Aciego, R., Álvarez, P., Muñoz, C. (2004). Análisis del rol profesional del psicopedagogo: una visión desde la práctica. Cultura y Educación, 1 (17), 35-52.

Barbour, R. (2013). Los grupos de discusión en investigación cualitativa. Madrid: Morata.

Domingo, J. (2012). Asesoramiento al centro educativo. Barcelona: Octaedro. 
Hernández, V., Santana, L. y Cruz, A. (2007). El asesoramiento de los Equipos de Orientación Educativa y Psicopedagógica (EOEP) en el contexto de los centros educativos: un estudio cualitativo. Revista de Investigación Educativa, 25(2), 287-304.

Santana, L. (2014). Orientación Educativa e Intervención Psicopedagógica. Madrid: Pirámide.

Stake, R. (2005). Investigación con estudio de casos. Madrid: Morata.

\section{Fuentes electrónicas}

Aguaded, $M^{\mathrm{a}}$ y Aguaded, J.I. (2011). El orientador educativo como imagen del progreso de la sociedad actual. Un estudio sobre sus procesos de formación profesional. Infancias en Imágenes, 1 (10), 32-44. Recuperado el 12 de noviembre de 2015, de http://rabida.uhu.es/dspace/bitstream/handle/10272/11280/El_orientador_educativo.pdf?seque nce $=4$

Álvarez, C. y San Fabián, J.L. (2012). La elección del estudio de caso en la investigación cualitativa. Gaceta de Antropología, 1 (28). Recuperado el 25 de Septiembre de 2015, de: http://www.ugr.es/ pwlac/G28_14Carmen_Alvarez-JoseLuis_SanFabian.html

Boza, A., Toscano, Ma. y Salas, M. (2007). ¿Qué es lo que hace un orientador? Roles y funciones del orientador en educación secundaria. En Clave Pedagógica, Revista Internacional de Investigación e Innovación educativa, 9, 111-131. Recuperado el 20 de Noviembre de 2015, de: http://www.uhu.es/publicaciones/ojs/index.php/xxi/article/view/520/756

CIDE. (2009). Orientación educativa: fundamentos teóricos, modelos institucionales y nuevas perspectivas. Madrid: Secretaría General Técnica. Recuperado el 16 de Diciembre de 2015, de: http://www.apega.org/attachments/article/379/orientacion_educativa.pdf

Calvo, A. Haya, I. y Susinos, T. (2012). El rol del orientador en la mejora escolar. Una investigación centrada en la voz del alumnado como elemento de cambio. Revista de Investigación en Educación, 10 (2), 7-20. Recuperado el 20 de noviembre de 2015, de http://reined.webs.uvigo.es/ojs/index.php/reined/article/viewFile/490/225

Cholewa, B., Goodman-Scott, E., Thomas, A. y Cook, J. (2017). Teachers' Perceptions and Experiences Consulting with School Counselors: A Qualitative Study. Professional School Counseling, 20 (1), 77-88. Recuperado el 20 de Febrero de 2017, de: http://professionalschoolcounseling.org/doi/10.5330/1096-2409-20.1.77?code=asca-site

Domingo, J. (2010a). Comprender y redireccionar las prácticas de asesoría. Revista Iberoamericana de Educación, (54), 65-83. Recuperado el 25 de Mayo de 2016, de: http://www.rieoei.org/rie54a03.pdf

Domingo, J. (2010b). Las Funciones del Orientador Educativo. Revista digital de la Universidad de Padres, (7). Recuperado el 22 de Noviembre de 2015, de: http://revista.universidaddepadres.es/index.php?option=com_poll\&view=poll\&id=15\&ltemid=35 0

González, I. y García, F. (2007). Elaboración de un perfil de la orientación en Educación Secundaria desde las demandas de la comunidad educativa. Revista Española de Orientación y Psicopedagogía, 2 (18), 215-227. Recuperado el 11 de Febrero de 2016 de: http://revistas.uned.es/index.php/reop/article/view/11313/pdf

Jiménez, I. (2012). La entrevista en la Investigación Cualitativa: Nuevas Tendencias y Retos. Revista Calidad en la Educación Superior, 119-139. Recuperado el 17 de Diciembre de 2015, de: http://dialnet.unirioja.es/servlet/articulo?codigo=3945773 
Lago, J. y Onrubia, J. (2011). Una estrategia general de asesoramiento para la mejora de la práctica educativa. Profesorado. Revista de currículum y formación del profesorado, 12(1). Recuperado el 12 de Enero de 2016, de: http://www.ugr.es/ recfpro/rev121COL5.pdf

Martínez, C., Krichesky, G. y García, A. (2010). El orientador escolar como agente de cambio interno. Revista Iberoamericana de Educación, 54, 107-122. Recuperado el 13 de febrero de 2016, de: http://rieoei.org/rie54a05.htm

Odegard-Koester, M. A. y Watkins, P. (2016). Collaborative Relationships between Principals and School Counselors: Facilitating a Model for Developing a Working Alliance. Journal of school Counseling, 14 (9). Recuperado el 3 de Marzo de 2018, de: https://eric.ed.gov/?q=school+counselor\&pg=2\&id=EJ1119083

Santana, L. (2010). La innovación educativa: un desafío para los orientadores como agentes promotores de las iniciativas de cambio. Revista Española de Orientación Psicopedagógica, 21(2), 261-270. Recuperado el 15 de Febrero de 2016,de: http://www.uned.es/reop/pdfs/2010/21-2\%20-\%20Lidia\%20E\%20Santana.pdf

Sink, C. A. (2016). Incorporating a Multi-Tiered System of Supports into School Counselor Preparation. The Professional Counselor, 6 (3) 203-219. Recuperado el 4 de Marzo de 2018, de: https://eric.ed.gov/?q=school+counselor\&id=EJ1115898

Vélaz de Medrano, C. (2008). Formación y profesionalización de los orientadores desde el enfoque de competencias. Educación XXI, 11, 155-181. Recuperado el 12 de Abril de 2016, de: http://revistas.uned.es/index.php/educacionXX1/article/view/313

Yáñez, J. (2008). Construir la relación de asesoramiento. Un enfoque institucional basado en la comunicación. Profesorado, Revista de currículum y formación del profesorado, 1 (12), 1-16. Recuperado el 22 de Enero de 2016, de http://www.redalyc.org/pdf/567/56717073015.pdf

Fecha de entrada: 20 Junio de 2016

Fecha de revisión: 8 Marzo de 2018 Fecha de aceptación: 20 Marzo de 2018 